\title{
In vitro screening of potato (Solanum tuberosum L.) cultivars for drought stress tolerance.
}

\author{
Labeb, R. K.*, H. E. M. Zaki ${ }^{12}$, Y. Y. Abdel-Ati ${ }^{1}$ and M. H. Zaky ${ }^{3}$ \\ ${ }^{1}$ Department of Horticulture, Faculty of Agriculture, Minia University, Minia 61519, Egypt. \\ ${ }^{2}$ college of Applied Sciences, Ministry of Higher education, Sultanate of Oman. \\ ${ }^{3}$ Horticulture Research Institute, Agriculture Research Center, Giza 12619, Egypt.
}

\begin{abstract}
This study was carried out at the tissue culture lab, Horticulture Department, Faculty of Agriculture, Minia University, Minia, Egypt. under in vitro condition to screening the response of four potato varieties (Diamond; Burren; Maritiema and Lady Balfour) to water stress induced by adding sorbitol in the culture medium. Four concentrations of sorbitol (0.0, 0.1, 0.2 and 0.3 mole /liter), in Murashige-Skoog (MS) medium were used for exerting the water deficiency stress on the plantlets with three replications, in completely randomized design. Data revealed that the potato cultivars were significant differed for plantlet height, number of leaves per plantlet, number of nodes per plantlet, number of roots per plantlet and plantlet weight in the medium without sorbitol. Moreover, different response of potato cultivars was observed to various levels of sorbitol concentrations. Increasing the concentration of sorbitol lead to significant decreased in all studied traits, as compared with control treatment (sorbitol free). However, the most reduction of morphological parameters was observed in Maritiema and Lady Balfour cultivars, and considered as susceptible cultivars for water deficit. These results indicated also that the Diamond variety is a droughttolerant than the other potato cultivars which used in the current study. These results indicated that the simulation of drought stress under in vitro conditions during the regeneration process constitutes a convenient way to study the effects of drought on potato responses. In-vitro this technique was shown to be useful in identifying relatively droughttolerant genotypes at early stages of development and this can be a very useful tool for screening large number of varieties or breeding lines of genotypes within a short time.
\end{abstract}

Keywords: Potato, Sorbitol, Water deficit, Plantlets, in Vitro, Varieties.

\section{Introduction}

Potato (Solanum tuberosum L.) is one of the most important vegetables in the world (Albiski et al., 2012). Potato is very sensitive to water-stress because of its shallower root system (Iwama and Yamaguchi, 2006). The identification of potato genotypes tolerant to abiotic stress is currently needed, since climate change

\footnotetext{
*Corresponding author: Remonda K. Labeb remondakhames@gmail.com;

Tel.: +01551273900

Received: February 6, 2020;

Accepted: March 31, 2020;

Published: April 2, 2020.
}

is associated with an increase in temperature of the planet and a decrease in precipitation (Lobell et al., 2011 and Barra et al., 2013). Therefore, the sustainability of production will depend on the identification and development of new drought tolerant varieties (Cochard et al., 2008, Rukundo, 2012). Moreover, Taiz and Zeiger (2006) reported that it is necessity to identify potato genotypes adapted to drought, which has become urgent because of the effect of this stress type on the growth and production of this crop. 
Gopal and Iwama (2007) reported that Potato genotypes differ in their tolerance to drought. Also, Levy (1986); Susnoschi and Shimshi (1985) found that there are differences in potato cultivars susceptibility to drought. Moreover, (Tican et al., 2016) showed also, that the sensitivity to drought was not uniform on potato varieties.

Drought is known to adversely effect on potato plant height (Lahlou and Ledent, 2005). Drought in potato lead to reduce the rate and duration of growth (Hang and Miller, 1986) and the number of leaves produced (Jefferies, 1993). Moreover, the major effects of water stress on potato plant are decreases in number of leaves, plant height, tuber growth, quality and yield as reported by (Arvin and Donnelly, 2008; Hassanpanah, 2009). Also, Tourneux et al. (2003) and Lahlou and Ledent (2005) mentioned that water-stress under potato field conditions is adversely affect plant growth including stem height. A growth inhibition is a common response to drought and osmotic stress in plants (Skirycz and Inzé, 2010). Drought leads to decreased tissue water content resulting in inhibited cell elongation (Taiz and Zeiger, 2006). Same conclusion was reported by Alfredo et al., (2004) who mentioned that the reduction in potato growth may be due to inhibition of plant cell division which results from water stress. Because a selection for drought tolerant genotypes in the field is time consuming, cost intensive and difficult to reproduce, it was proposed that Screening genotypes for their response to stress conditions in vitro might be an alternative to efficiently check material for its reaction to osmotic stress (Gopal and Iwama, 2007). Various in vitro methods to induce water stress in plants have been described, which use chemical agents that reduce water potential in the culture medium (Barra et al., 2013) such as sorbitol.

Sorbitol, a six-carbon sugar alcohol, is one of the most frequently found polyols in plants. It is a direct product of photosynthesis in mature leaves, in parallel with sucrose, whereas both serve similar functions, such as translocation of carbon skeletons and energy between sources and sink organs (Jain et al., 2010). Previous studies by Gopal and Iwama (2007) and Gopal et al. (2008) simulated drought stress condition in vitro by the addition of sorbitol to solid MS medium. This resulted in the identification of $0.2 \mathrm{M}$ sorbitol to give the best response of the potato genotypes to distinguish more tolerant ones among the test set. Osmotic stress was chosen as it is one of the factors contributing to drought stress. Tican et al. (2016) reported that sorbitol has been used by several workers as osmotic stress agents for in vitro selection. They mentioned also that the increasing amount of sorbitol, water absorption becomes difficult for plantlets from nutrient medium and thus was simulate the effect of drought over microplants. They added that with the increasing amount of sorbitol into the growth medium, the drought intensity was bigger that lead to decrease the number of internodes on potato plantlets. They found that potato genotypes were differed in their response to water stress by applying sorbitol on nutritive medium.

Gopal and Iwama (2007) mentioned that some researchers reported that addition of sorbitol on Murashige-Skoog medium decreased water potential, inducing drought stress affecting shoot and root growth. They added that these similarities 
in the effects of water-stress under in vitro and in vivo conditions suggest that the in vitro system can be used as an alternative to field evaluations for studying the general effect of water-stress on plant growth and development. They mentioned that the addition of sorbitol to the MS medium decreased the water potential of the media inducing water-stress that adversely affected both shoot and root growth of the plantlets. Also, under extreme water-stress conditions as induced by $0.4 \mathrm{M}$ sorbitol, all genotypes had little shoot as well as root growth. Albiski et al. (2012) reported that sorbitol inducing water stress in morphological parameters in potato. They mentioned that the drought induced by addition of sorbitol on MS medium led to decrease in the potato stem length, number of leaves and number of roots per plant according to potato genotypes and drought.

The objective of this study is to determine the response of four potato genotypes to water stress, by measuring the morphological characteristics associated with the vegetative growth of potato plantlets in vitro using sorbitol as a water stress inductor. In order to develop a practical and effective method in vitro screening of potato, which may provide a system for effectively differentiating the genotypes for their expected root mass production under field conditions.

\section{Materials and Methods}

The present study was performed at Laboratory of Horticulture Department, Faculty of Agriculture, El- Minia University, El-Minia, Egypt from 2016 to 2019.

Plant material Four potato (Solanum tuberosum L.) cultivars; Burren, Diamond,
Lady Balfour and Maritiema were used for this study. Tuber seeds of all genotypes were obtained from Agricultural Research Center, Horticulture Department, Mallawi, El-Minia, Egypt.

\section{Treatment of sorbitol}

Three levels of sorbitol $(0.1,0.2$ and 0.3 mole /liter) as a water-stress were added to the MS medium and compared with control (sorbitol free or 0.0 mole/l).

\section{In Vitro propagation}

A tissue culture method was used for the rapid micropropagation of the previously mentioned potato cultivars. About 50 plantlets were regenerated, in a sequential procedure, from a single isolated shoot tip. Shoot tips were surface sterilized with $0.25 \%$ calcium hypochlorite for 5 minutes and rinsed thoroughly with sterile distilled water. Thereafter, the surface sterilized shoot tips were cultured Murashige and Skoog (MS) basic medium as reported by Murashige and Skooge (1962). The pH was adjusted to $5.7 \pm 0.1$ before the addition of Agar and subsequent autoclaving at $121^{\circ} \mathrm{C}$ and 15 psi for $20 \mathrm{~min}$. Tissue excision, implantation and transfer procedure were carried out under sterile conditions. The cultured shoot tips were incubated for four weeks in the incubation room at $25 \pm 2 \mathrm{oC}$ with $16: 8 \mathrm{~h}$ photoperiod and $2000-4000$ lux, as a light intensity.

\section{Drought treatment}

Individual stem nodes were transferred to jars containing $20 \mathrm{ml}$ of MS growth medium, 30 g. $1^{-1}$ sucrose and 30 g. . $^{-1}$ Agar supplemented with sorbitol, as a waterstress, of $0,0.1,0.2$ or 0.3 mole/liter at $\mathrm{pH}$ 5.7 and allowed to grow for 4 weeks to test their tolerance or sensitivity to drought. Hundred plantlets per cultivar were evaluated for each of the four drought 
treatments and the experiment was repeated at approximately monthly intervals three times. All subcultures were maintained under $25 \pm 20^{\circ} \mathrm{C}$ with $16: 8 \mathrm{~h}$ photoperiod and 2000-4000 lux, as a light intensity. Growth and morphological changes due to drought stress in culture were observed and recorded.

\section{Recorded data}

When subcultures were 30 days old (without sub culturing) and fully-grown having stout stems and broad leaves in the control treatment (i.e., MS medium without sorbitol) or medium with sorbitol as a water stress, data were recorded for various shoot and root characteristics as the following:

a) Plantlet height $(\mathrm{cm})$ : this was determined at measured as the length of the main stem from base to the shoot tip.

b) Number of plantlet leaves.

c) Number of plantlet roots.

d) Plantlet fresh weight (g).

\section{Statistical analysis}

The experimental design was a $4 \times 4$ (cultivars $\times$ Sorbitol concentrations) factorial experiment in a randomized complete block with three replications.

The data were statistically analyzed using ANOVA analysis to determine the level of significant differences between treatments means as compared to the control at $\mathrm{P} \leq$ 0.05 level of significance Gomez and Gomez (1984).

\section{Results and Discussion}

\section{Plantlet height}

The effects of drought on the plantlet height grown in vitro from shoot apices are shown in in Table 1. drought decreased plantlet height in all potato cultivars, the most reduction obtained by Maritiema and Lady Balfour (52.6 and 38.34\%), respectively. drought decreased plantlet height in all potato cultivars, the most reduction obtained by Maritiema and Lady Balfour (52.6 and 38.34\%), respectively. In general, all of potato cultivars showed more plantlet height at the control level (sorbitol free), all of the cultivars significantly decreased expect Diamond cultivar did not differ significantly and had the higher plantlets height $(11.67 \mathrm{~cm})$. Similar conclusions were reported by Gopal and Iwama (2007) and Albiski et al. (2012) who reported that addition of sorbitol on MS medium decreased the water potential and inducing drought stress affecting shoot growth. The reduction in plantlet height may be due to that with increasing amount of sorbitol, water absorption becomes difficult for plantlets from nutrient medium and thus simulates the effect of drought on microplants pointed out Tican et al. (2016). Similar conclusions were reported by (Tourneux et al.,2003; Lahlou and Ledent, 2005; Arvin and Donnelly, 2008; Hassanpanah, 2009; Albiski et al., 2012) their studies revealed that the water stress decreased plant height of potato plants. Also, drought decreased water content in tissues of plants, resulting in inhibited cell elongation as reported by (Alfredo et al.,2004; Taiz and Zeiger,2006). Same conclusion was reported by Alves and Setter (2004) who mentioned that the reduction in potato growth may be due to inhibition of plant cell division which results from water stress.

The result indicated also that Diamond cultivar is a drought tolerant cultivar. These results agree with (Susnoschi and Shimshi, 1985; Levy,1986; Gopal and 
Iwama, 2007; Albiski et al., 2012; Tican et al., 2016).

\section{Number of plantlet leaves}

Data in (Table 1) revealed that the potato Cultivars (Burren, Diamond, Lady Balfour and Maritiema) were significant differed for number of leaves per plantlet in the medium without sorbitol. Significant decrease in number of leaves for potato Cultivars used are observed with increasing sorbitol concentration from 0.2 to 0.3 mole / liter in MS medium than those in without or lower sorbitol concentrations. Also, the decrease in number of leaves for potato Cultivars are more pronounced in Maritiema and Lady Balfour varieties with no significant between them at 0.3 mole / liter sorbitol as compare to Diamond and Burren cultivars. The reduction in number of leaves may be due to increasing amount of sorbitol, water absorption becomes difficult for plantlets from nutrient medium and thus simulates the effect of drought on microplants as reported by Andreea et al. (2016). Moreover, the effect of sorbitol is reported by several authors who stated that the sorbitol has been used as osmotic stress agents for in vitro to inducing drought stress that affecting on shoot growth Gopal and Iwama (2007). These results are in agreement with those reported by (Jefferies, 1993; Arvin and Donnelly, 2008; Hassanpanah, 2009) who found that the major effects of water stress on potato plant growth such as decreasing number of leaves. Data revealed also that the Diamond potato variety have high drought tolerant cultivar than the other potato cultivar used due to have forming higher number of leaves while, Maritiema and Lady Balfour cultivar are considered as a drought sensitive cultivar by forming lower number of leaves. This result indicates different response of potato genotypes to various levels of sorbitol concentrations or drought stress. These results are agreed with those obtained by (Susnoschi and Shimshi, 1985; Levy, 1986; Gopal and Iwama, 2007; Albiski et al., 2012; Tican et al., 2016) who reported that potato genotypes differed in their tolerance to drought stress.

Table 1. Performance of potato genotypes at different concentrations of Sorbitol for various characters in vitro

\begin{tabular}{clcccc}
\hline \multirow{2}{*}{ Character } & \multirow{2}{*}{ Genotype } & \multicolumn{4}{c}{ Sorbitol Concentration (mole / liter) } \\
\cline { 3 - 6 } & & 0.0 & 0.1 & 0.2 & 0.3 \\
\hline Plantlet height $(\mathrm{cm})$ & Diamond & $11.67^{\mathrm{a}}$ & $11.67^{\mathrm{a}}$ & $11.33^{\mathrm{a}}$ & $11.33^{\mathrm{a}}$ \\
& Burren & $9.00^{\mathrm{b}}$ & $9.00^{\mathrm{b}}$ & $8.33^{\mathrm{c}}$ & $8.00^{\mathrm{c}}$ \\
& Maritiema & $6.33^{\mathrm{d}}$ & $6.33^{\mathrm{d}}$ & $4.33^{\mathrm{e}}$ & $3.00^{\mathrm{f}}$ \\
& Lady Balfour & $4.33^{\mathrm{e}}$ & $4.00^{\mathrm{e}}$ & $4.00^{\mathrm{e}}$ & $2.67^{\mathrm{f}}$ \\
\hline LSD 5\% & & \multicolumn{4}{c}{} \\
\hline Number of plantlet leaves & Diamond & $12.67^{\mathrm{a}}$ & $12.00^{\mathrm{a}}$ & $11.00^{\mathrm{b}}$ & $10.67^{\mathrm{bc}}$ \\
& Burren & $10.00^{\mathrm{cd}}$ & $10.00^{\mathrm{cd}}$ & $9.67^{\mathrm{d}}$ & $8.67^{\mathrm{e}}$ \\
& Maritiema & $8.67^{\mathrm{e}}$ & $7.67^{\mathrm{f}}$ & $7.33^{\mathrm{f}}$ & $4.33^{\mathrm{h}}$ \\
& Lady Balfour & $6.33^{\mathrm{a}}$ & $6.33^{\mathrm{g}}$ & $5.67^{\mathrm{g}}$ & $3.67^{\mathrm{h}}$ \\
& & \multicolumn{3}{c}{0.77} & \\
\hline
\end{tabular}

Different letters between cultivars indicate significant differences on a global significance level of $5 \%$. 


\section{Number of plantlet node}

Data revealed that the number of nodes per plantlet in the medium without sorbitol was significantly decreased for all potato cultivars under study conditions (Table 2). Increasing sorbitol concentration from 0.2 to 0.3 mole / liter in MS medium, decreased number of nods per plantlet than those in lower sorbitol concentrations. Also, the decrease in number of nodes for potato are more pronounce in Maritiema and Lady Balfour cultivars. With significant between them at 0.3 mole / liter sorbitol as compare to Diamond and Burren. This result is agreed with Tican et al. (2016) who found that with the increasing amount of sorbitol into the growth medium, the drought intensity was bigger that lead to decreasing the number of internodes on potato plantlets. The reduction of nodes number may be due to the drought as reported by (Arvin and Donnelly, 2008; Hassanpanah, 2009) which lead to decrease tissue water content resulting in inhibited of cell elongation (Taiz and Zeiger, 2006). The use of sorbitol as chemical agents to reduce water potential in the culture medium was reported by (Noiraud et al., 2001; Barra et al., 2013: Tican et al., 2016) which affecting on plant growth of potato. Moreover, data revealed that Diamond is a drought-tolerant cultivar than the other potato cultivars which used, due to have forming higher number of nodes while, Maritiema and Lady Balfour cultivars are considered as a drought sensitive cultivars by forming lower number of nodes. This result revealed different response of potato to various levels of sorbitol concentrations. These results are similar obtained by (Susnoschi and Shimshi, 1985; Levy, 1986; Gopal and Iwama, 2007; Albiski et al., 2012; Tican et al., 2016) who reported that potato genotypes differed in their tolerance to drought stress.

The effects of sorbitol were reported by several authors, they stated that the sorbitol has been used as osmotic stress agents for in vitro to inducing drought stress that affecting on shoot growth (Gopal and Iwama, 2007) or morphological parameters (Albiski et al., 2012).

\section{Number of plantlet roots}

Data that the potato are significant differ for number of roots per plantlet in the medium without sorbitol (Table 2). Roots number of Burren, Maritiema and Lady Balfour are significant decrease with increasing sorbitol concentration in medium than those in without sorbitol. However, the decrease in roots number are more pronounce in Maritiema and Lady Balfour with significant between them at 0.3 mole / liter sorbitol as compared to Diamond and Burren.

Similar results were reported by Albiski et al., (2012) who reported that the increasing of sorbitol concentration in MS medium decreased the root number of potato due to the drought. Also, (Tourneux et al.,2003; Lahlou and Ledent, 2005) who mentioned that water-stress is adversely effects on potato root number. The use of sorbitol as chemical agents to reduce water potential in the culture medium was reported by (Noiraud et al., 2001; Barra et al., 2013; Tican et al., 2016) which affecting on plant growth of potato. Also, Gopal and Iwama (2007) who found that the addition of sorbitol to the MS medium decreased the water potential of the media inducing water-stress that adversely 
affected both shoot and root growth of the plantlets.

Data in revealed that Diamond cultivar does not differ significantly for plantlet root number, with increasing sorbitol concentration in medium. This result indicates that Diamond is a droughttolerant than the other potato varieties used. Moreover, the data show that Maritiema and Lady Balfour are considered as a drought sensitive by forming lower number of roots with increasing sorbitol concentration in medium. Similar results were reported by (Susnoschi and Shimshi, 1985; Levy, 1986; Gopal and Iwama, 2007; Albiski et al., 2012; Tican et al., 2016) this result agree with Albiski et al. (2012).

\section{Plantlet weight}

Data revealed that the potato genotypes are significant differ for plantlet weight in the medium without sorbitol Significant decrease in plantlet weight for potato observed with increasing sorbitol concentration from 0.2 to 0.3 mole $/ 1$ in MS medium than those in lower sorbitol concentrations. Also, the decrease in plantlet weight for potato are more pronounce in Maritiema and Lady Balfour with significant between them at 0.3 mole / liter sorbitol as compare to Diamond and Burren. The reduction in plantlet weight may be due to that with increasing amount of sorbitol in medium which lead to lower the plantlets height, number of leaves, number of nodes, as well as the number of roots. Similar results were reported by Albiski et al. (2012).

Table 2. Performance of potato genotypes at different concentrations of Sorbitol for various characters in vitro.

\begin{tabular}{clcccc}
\hline \multirow{2}{*}{ Character } & \multirow{2}{*}{ Genotype } & \multicolumn{4}{c}{ Sorbitol Concentration (mole / liter) } \\
\cline { 3 - 6 } & & 0.0 & 0.1 & 0.2 & 0.3 \\
\hline Number of plantlet nodes & Diamond & $10.33^{\mathrm{a}}$ & $9.00^{\mathrm{b}}$ & $9.00^{\mathrm{b}}$ & $8.33^{\mathrm{c}}$ \\
& Burren & $8.00^{\mathrm{c}}$ & $8.00^{\mathrm{c}}$ & $8.00^{\mathrm{c}}$ & $6.67^{\mathrm{d}}$ \\
& Maritiema & $6.67^{\mathrm{d}}$ & $6.00^{\mathrm{e}}$ & $5.67^{\mathrm{e}}$ & $2.33^{\mathrm{g}}$ \\
& Lady Balfour & $4.33^{\mathrm{f}}$ & $4.33^{\mathrm{f}}$ & $2.67^{\mathrm{g}}$ & $1.67^{\mathrm{h}}$ \\
\hline LSD 5\% & & \multicolumn{5}{c}{0.57} \\
\hline Number of plantlet roots & Diamond & $18.00 \mathrm{a}$ & $17.67 \mathrm{a}$ & $17.67 \mathrm{a}$ & $17.33 \mathrm{a}$ \\
& Burren & $15.00 \mathrm{a}$ & $14.33 \mathrm{a}$ & $12.00 \mathrm{c}$ & $11.00 \mathrm{de}$ \\
& Maritiema & $12.33 \mathrm{c}$ & $11.67 \mathrm{~cd}$ & $10.33 \mathrm{ef}$ & $3.67 \mathrm{j}$ \\
& Lady Balfour & $10.00 \mathrm{f}$ & $7.67 \mathrm{~g}$ & $6.00 \mathrm{~h}$ & $5.00 \mathrm{i}$ \\
\hline LSD 5\% & & \multicolumn{5}{c}{0.71} & \\
\hline Plantlet weight (gm) & Diamond & $0.57^{\mathrm{a}}$ & $0.57^{\mathrm{a}}$ & $0.49^{\mathrm{b}}$ & $0.43^{\mathrm{bc}}$ \\
& Burren & $0.45^{\mathrm{b}}$ & $0.43^{\mathrm{bc}}$ & $0.37^{\mathrm{cd}}$ & $0.36^{\mathrm{d}}$ \\
& Maritiema & $0.25^{\mathrm{e}}$ & $0.24^{\mathrm{e}}$ & $0.15^{\mathrm{f}}$ & $0.15^{\mathrm{f}}$ \\
& Lady Balfour & $0.21^{\mathrm{e}}$ & $0.14^{\mathrm{f}}$ & $0.13^{\mathrm{f}}$ & $0.06^{\mathrm{g}}$ \\
\hline LSD 5\% & & \multicolumn{5}{c}{0.06} & \\
\hline
\end{tabular}

Different letters between cultivars indicate significant differences on a global significance level of $5 \%$. 
This result revealed different response of potato to various levels of sorbitol concentrations. These results are with (Susnoschi and Shimshi, 1985; Levy, 1986; Gopal and Iwama, 2007; Albiski et al., 2012; Tican et al., 2016) who reported that potato genotypes differed in their tolerance to drought stress. The effect of sorbitol are reported by several authors who stated that the sorbitol has been used as osmotic stress agents for in vitro to inducing drought stress that affecting on morphological parameters as reported by (Gopal and Iwama, 2007; Gopal et al., 2008; Albiski et al., 2012).

\section{Conclusion}

These results show significant variations among the potato for drought tolerance based on morphological parameters under in vitro condition. Higher concentration of sorbitol 0.3 mole/liter severely effect on plantlet height, number of plantlet leaves, number of plantlet nodes, number of plantlet roots and plantlet weight of the diamond, Burren, Maritiema and lady Balfour potato. Moreover, these results indicate that sorbitol can been used to stimulate drought stress in vitro for identifying relatively potato droughttolerant genotypes at early stages of development and this can be a very useful tool for screening large number of varieties or breeding lines of genotypes in potato plants.

\section{References}

Albiski, F., Najla, S., Sanoubar, R., Alkabani, N. and Murshed, R. (2012) 'In vitro screening of potato lines for drought tolerance', Physiol. Mol. Biol. Plants, 18(4), pp. 315-321. doi: $10.1007 / \mathrm{s} 12298-012-0127-5$
Alfredo. Alves A. C and Tim setter, L. (2004). 'Response of Cassava Leaf Area Expansion to Water Deficit: Cell Proliferation', Cell Expansion and Delayed Development Annals of Botany 2008 , doi:10.1093/aob/mch179

Tican, A., Cioloca, M., Chiru, N. and Bădărău, C. (2016) 'Influence of different in vitro simulators for hydric stress for growth and development of potato', Scientific Bulletin. Series F. Biotechnologies, Vol. XX, ISSN 2285-1364, pp.105-112.

Arvin, M.J. and Donnelly, D.J. (2008). Screening potato cultivars and wild species to abiotic stresses using an electrolyte leakage bioassay'. Journal of Agricultural Science and Technology 10, pp. 33-42.

Barra, M., Correa, J., Salazar, E. and Sagredo, B. (2013). 'Response of potato (Solanum tuberosum L.) germplasm to water stress under in vitro conditions'. American Journal of Potato Research, 90, pp.591-606. doi: 10.1007/s12230-013-9333-0

Cochard, H., Barigah, S., Kleinhentz, M., Eshel, A. (2008). 'Is xylem cavitation resistance a relevant criterion for screening drought resistance among Prunus species? 'Journal of Plant Physiology, 165, pp.976-982. doi: 10.1016/j.jplph.2007.07.020

Gomez, K.A. and A.A. Gomez, (1984). 'Statistical procedures for agricultural research $\left(2^{\mathrm{Ed} .}\right)$ '. John wiley and sons, NewYork, 680p.

Gopal, J. and Iwama, K. (2007). 'In vitro screening of potato against waterstress mediated through sorbitol and polyethylene glycol'. Plant Cell Rep., 26, pp. 693-700. doi: 10.1007/s00299006-0275-6

Gopal, J., Iwama K. and Jitsuyama, Y. (2008). 'Effect of water stress mediated through agar on in vitro growth of potato'. In Vitro Cellular \& Developmental Biology Plant, 44, 
pp.221-228. doi: 10.1007/s11627-0079102-1

Hang, A.N. and D.E. Miller. (1986). 'Yield and physiological responses of potatoes to deficit, high frequency sprinkler irrigation'. Agron. J., 78, pp. 436-440. doi: 10.2134/agronj1986.00021962007800 030008x

Hassanpanah, D. (2009). 'In vitro and in vivo screening of potato cultivars against water stress by polyethylene glycol and potassium humate'. Biotechnology, Asian Network for Scientific Information pp.1-6. doi: 10.3923/biotech.2009.132.137

Iwama, K. and Yamaguchi, J. (2006) 'Abiotic stresses'. In: Gopal J, Khurana SM Paul (Eds) Handbook of potato production, improvement and postharvest management. Food Product Press, New York, pp 231-278.

Jain, M., Tiwary S., Gadre, R. (2010). 'Sorbitol-induced changes in various growth and biochemical parameters in maize'. Plant Soil and Environment, 6, pp.263-267. doi: 10.17221/233/2009PSE

Jefferies, R.A. (1993). 'Responses of potato genotypes to drought. 11. Leaf area index, growth and yield'. Annals Applied Biol. 122 (1), pp. 105-1 12. doi:

$10.1111 / \mathrm{j} .1744-$ 7348.1993.tb04018.x

Lahlou, O. and Ledent, J.F. (2005) ' Root mass and depth, stolons and roots formed on stolons in four cultivars of potato under water stress'. Eur. J. Agron., 22, pp.159-173. doi: 10.1016/j.eja.2004.02.004

Levy, D. (1986) 'genotypic variation in the response of potatoes (Solanum tuberosum L.) to high ambient temperatures and water deficit'. Field Crop Res., 15, pp.85-96. doi: 10.1016/0378-4290(86)90103-6

Lobell DB, Schlenker W. and CostaRoberts, J. (2011). 'Climate trends and global crop production since 1980', Science 333(6042), pp.616-620. doi: 10.1126/science. 1204531

Murashige, T. and Skooge, F. (1962) 'A revised medium for rapid growth and bioassay with tobacco tissue', Physiology Planta,15, pp. 473-497. doi: $\quad 10.1111 /$ j.13993054.1962.tb08052.x

Noiraud N., Maurousset L. and Lemoine R. (2001) 'Transport of polyols in higher plants', Plant Physiology and Biochemistry, 39, pp.717-728. doi: 10.1016/S0981-9428(01)01292-X

Rukundo, P., Carpentier, S.C. and Swennen, R. (2012) 'Development of in vitro technique to screen for drought tolerant banana varieties by sorbitol induced osmotic stress', African Journal of Plant Science 6(15), pp.416-425. doi: 10.5897/AJPS12.101

Skirycz, A., and Inze, D. (2010) ' More from less: plant growthunder limited water', Curr. Opin. Biotechnol., 21, 197-203. doi: 10.1016/j.copbio.2010.03.002

Susnoschi, M., Shimshi, D. (1985) Growth and yield studies of potatodevelopment in a semi-arid region'. 2. Effect of water stress and amounts of nitrogen top dressing on growth of several cultivars. Potato Res., 28, pp.161-176 doi: 10.1007/BF02357442

Taiz, L., Zeiger, E. (2006) 'Plant physiology, $4^{\text {th }}$ ed', Sinauer Associates Inc, Sunderland, Massachusetts.

Tourneux, C., Devaux, A., Camacho, M.R., Mamani, P., Ledent, J.F. (2003) ' Effects of water shortage on six potato genotypes in the highlands of Bolivia (I): morphological parameters, growth and yield', Agronomie 23, pp.169-179 doi: 10.1051/agro:2002079 